\title{
Pathways to parenting understanding addiction incarceration and parenting: a pilot investigation
}

\begin{abstract}
Background: Using a multi method approach which involved the participants filling out questionnaires and engaging in focus group discussions this study explored the parenting challenges, perceptions of social support and re-entry preparation of currently incarcerated, alcohol or drug dependent mothers (AOD) preparing to reenter back into their parenting role, family life and their communities. The sample consisted of 9 incarcerated mothers residing in a department of corrections residential substance abuse treatment center.

Findings: The findings suggest that these mothers report having adequate family and personal resources, and rate their parenting interactions with their children as positive. However results from the focus group data reveal that they express concern with specific parenting issues (i.e. parenting children on medication), concern with family support upon reentry (i.e. fragile family support) and dissatisfaction with their preparation to reenter into their families and communities (i.e. lack of job training and employment support). We offer the following suggestions for those working with this population of mothers. Pathways to parenting may differ for recovering substance abusing mother than for other formerly incarcerated mothers. Second, drug rehabilitation transition programs must be structured to provide targeted parenting support and deal with real life issues and concerns that may occur post release.
\end{abstract}

Keywords: parenting, addiction, frame work analysis, incarceration
Volume I Issue 2 - 2014

Yvette R Harris, Vanessa A Harris

Department of Psychology, Miami University, USA

Correspondence: Yvette R Harris, Department of Psychology, 90 North Patterson Avenue, Miami University, Oxford, Oho 45056, USA, Tel 513-529-2009, Email harrisyr@maimioh.edu

Received: April 22, 2014 | Published: June II, 20I4

\section{Introduction}

Concerned with the growing epidemic of alcohol and drug abuse among women, social scientists turned their attention to investigating the proximal and distal causes and consequences of their drug and alcohol use. ${ }^{1,2}$ As a result of this attention, a corpus of scholarship emerged exploring gender issues and drug abuse, ${ }^{3}$ gender issues and treatment, ${ }^{4}$ mothering and drug use ${ }^{5}$ and the influence of maternal drug use on child outcomes. ${ }^{6}$ Five general conclusions can be drawn from this research. First, unlike alcohol or drug dependent (AOD) men, many AOD dependent women have a history of childhood sexual and physical abuse. ${ }^{7}$ Second, in contrast to AOD dependent men, a number have untreated chronic mental and physical health problems. ${ }^{4}$ Third, there is need for substance abuse intervention programs tailored to the unique physical, emotional and relational needs of women. ${ }^{8} \mathrm{Critics}^{9,10}$ argue that current intervention programs are often modeled after programs designed for men and as such are ineffective in meeting and treating the needs of substance abusing women. Fourth, mothering in the midst of addiction and recovery present a host of challenges for parenting women. ${ }^{3}$ Fifth, there are physical, emotional, behavioral and intellectual consequences for children born to substance abusing mothers, ${ }^{11}$ however long term adverse outcomes maybe be buffered by social support, and early maternal drug intervention during pregnancy. ${ }^{12}$

Yet absent from this literature is a discussion of mothers who are AOD dependent -in recovery- incarcerated and preparing to re-enter back into the lives of their children and families from prison. These mothers represent a distinctively different subset of AOD dependent mothers in the following ways. First, unlike AOD dependent mothers who have not been incarcerated, incarceration brings on a host of life altering changes. ${ }^{13}$ That is, depending on the conditions of their release from prison they may have continued contact with the courts, and due their conviction may have limited employment, housing and government assistance options. Second, they must deal with the social stigma and psychological trauma of incarceration all of which serve to impact their children's adjustment to their incarceration and reentry and impact their parenting upon release. Third, most of these mothers express their desire to return to their parenting role upon re-entry and as such may have to renegotiate their parenting role or adjust to a peripheral parenting role all while maintaining sobriety. ${ }^{14}$

Given their challenges upon re-entry it is important for social scientists to begin to identify from the mother's perspectives and voices, their perceived and anticipated parenting challenges, their access to and identification of family support, social support and personal resources, and their reentry preparation as they transition back into the lives of children, their families and their communities.

Thus the goal of this exploratory project was three fold. One, to determine the parenting challenges and parenting goals of incarcerated AOD dependent mothers in recovery preparing to re-enter. Two, to obtain information on their social support, family and personal resources and their evaluation of the quality of those resources. Three, to determine how they are preparing for re-entry and their perceptions of the effectiveness of their re-entry preparation.

Our research was guided by the following specific questions.

a. What are the perceived and anticipated parenting challenges and goals of incarcerated mothers recovering from substance abuse and preparing to re-enter?

b. What is their evaluation of their family support, family resources and personal resources and their evaluation of the quality of those resources?

c. How are they preparing to re-entry, and what is their evaluation of the reentry services provided by their current correctional facility? 


\section{Method}

\section{Participants}

Nine mothers currently incarcerated in a department of corrections facility were recruited to participate in the investigation. The mothers were on average 37 years of age and divorced $(n=4)$, single $(n=2)$ and in a significant relationship $(\mathrm{n}=3)$. Four identified as African American; four identified as Caucasian; and one mother identified as other. The mean age of their children was ten years of age, (range 1-19). Four of their children had some type of disability; three of their children were labeled as having a behavior problem. These disabilities/problems ranged from Attention Deficit Hyperactive Disorder, Bipolar Disorder to learning disabilities and severe asthma. The majority of the mothers reported their children's fathers as the primary caretakers. In terms of education, three mothers had completed high school; three completed some high school, and one completed only junior high. Two indicated they had received specialized training.

The mothers were administered a series of questionnaires and participated in a 90minutes focus group session to further probe them about their parenting issues, challenges and goals upon release as well as their family and social support and their re-entry preparation. Our rationale for employing a multi method approach is based primarily on the recent consensus among social scientists stating that when the goal of the phenomenon under study is to yield direction for designing prevention and intervention programs, a multi method approach maybe more effective than employing a qualitative or quantitative approach alone. ${ }^{15}$ Furthermore, we felt that this type of design might be better suited to address our basic research questions for the study and to assist us in resolving discrepancies in the questionnaire data should any arise. The research was conducted at the facility and the mothers were offered a gift card for their participation in the research.

\section{Measures}

\section{Demographic questionnaire (DQ)}

Is a 30 item investigator created questionnaire designed to obtain information on marital status, educational information, mental health and physical health, incarceration history, personal physical abuse or sexual abuse, information on children, treatment during incarceration, and involvement in parenting programs while incarcerated.

\section{Center for epidemiological studies depression scale (CES-D)}

The CES-D is a 20 item short self-report scale designed to assess how frequently a person has experienced symptoms of depression during the past week. Respondents are requested to respond to such questions as "my sleep is restless", "I felt lonely", on a three point Likert type scale with 0 indicating rarely and 3 indicating all of the time. Total scores are tabulated with scores of 16 or higher suggesting depression. Scores range from 1-53.

\section{Parenting stress scale (PSS)}

Is an 18 item scale which assesses stress associated with different aspects of parenting. Respondents are requested to respond to such items as" I am happy in my role as a parent" on a five point Likert type scale with 1 indicating that they strongly disagree with the item and 5 indicting they strongly agree. Scores range from 18-90, with high scores suggesting higher level of parental stress.

\section{Parental relationship questionnaire (PRS)}

Is an 11 self-report scale that examines from the parents' perspective the parent child relationship in the following domains: attachment, communication, discipline practice, involvement, parenting confidence, satisfaction with child's school and relationship frustration? Respondents are asked to respond to such questions as "my child enjoys spending time with me", on a four point Likert type scale with 0 indicting that the statement never describes their beliefs about their relationship with their child or with 3 indicating that the statement almost always describes their beliefs about their experiences with their child. Scores below 40 indicate problems in this area.

\section{Family resource scale (FRS)}

Is a 30 item scale that assesses the adequacy of a family's tangible and intangible resources? Respondents are asked to indicate on a five point Likert type scale with 1 indicating not at all adequate, and 5 almost always adequate to such items as "to what extent are the following resources adequate for you and your family : Food for two meals a day, Dependable transportation. "Scores fall between 0 and 150 with higher scores suggesting more family resources.

\section{Personal resource questionnaire (PQ85)}

Is a 35 item scale which assesses perceived social support. Respondents are required to answer such questions as "there is someone I feel close to who makes me feel secure ", on a seven point Likert type scale, with 1 indicating that they strongly agree with the item and 7 indicating that they strongly disagree with the item. Scores range from 25-175 with higher scores suggesting greater levels of social support.

\section{Focus group questions}

Questions were designed to tap their perceptions of their parentchild relationship (e.g. what are their challenges? what are their goals?); their perceptions of their social support while incarcerated and upon re-entry (e.g. who do you receive support from?) and their preparation for re-entry (e.g. how are you preparing for re-entry? do you think the programs they offer while incarcerated prepare you for reentry?).

\section{Results}

The results of the demographic questionnaire are presented below.

\section{Mental health and physical health problems}

Seven of the participants reported being diagnosed with such mental health problems as depression, bipolar disorder, obsessive compulsive disorder, and schizophrenia. Six of the participants indicated that they received treatment for their mental health problems prior to incarceration (medication, individual counseling, and group counseling). Currently, all nine participants indicated receiving medication for their mental health problems. Four participants indicated experiencing various physical health problems ranging from diabetes, hypertension to arthritis.

\section{Criminal history}

The majority of the mothers reported committing felony 5 crimes; once incarcerated their sentence lengths varied from 30 days to 3 years.

\section{Abuse history}

The mothers indicated that they had experienced some form of abuse during childhood. With five experiencing physical abuse during childhood and three experiencing sexual abuse. Many reported that the abuse began during the school age period (6-9). 
The means for the questionnaires are presented in (Table 1). As the data in the table illustrate, the mother's CES-D scores were slight above average $(\mathrm{M}=17 ; \mathrm{SD}=12.6)$, suggesting that they were experiencing mild depression. The mother's responses to the Parental Stress questionnaire indicate they experience little parenting stress $(\mathrm{M}=35$; $\mathrm{SD}=11.3$ ); and their responses to the Family Resource questionnaire describe their family resources $(\mathrm{M}=111, \mathrm{SD}=28.5)$ as adequate. Their responses to the Personnel Resource questionnaire indicate that they rate the availability of a support system $(\mathrm{M}=131, \mathrm{SD}=31.5)$ as adequate as well. Their scores on the Parenting Relationship Questionnaire also suggest that they perceive their relationship with their children in the areas of Attachment $(\mathrm{M}-58, \mathrm{SD}=9.5)$, Communication $(\mathrm{M}=55$, $\mathrm{SD}=10.9)$, Discipline Practice $(\mathrm{M}=54, \mathrm{SD}=8.8)$, Involvement $(\mathrm{M}=61)$, $\mathrm{SD}=13.9)$, Parenting Confidence $(\mathrm{M}=53, \mathrm{SD}=9.0)$ as strong. They indicate Satisfaction with their Children's School $(\mathrm{M}=57,11.3)$, and experience low to minimal relationship frustration with their children $(\mathrm{M}=46, \mathrm{SD}=8.2)$.

Table I Means for the quantitative measures

\begin{tabular}{llll}
\hline Measures & Mean & SD & Range \\
\hline CESD & 17.7 & 12.6 & Mar-40 \\
Parental Stress (PSS) & 35 & 11.3 & $20-55$ \\
Family Resource (FRS) & $1 \mathrm{II}$ & 28.5 & $58-146$ \\
Personal Resource (PQ85) & $13 \mathrm{I}$ & 31.5 & $81-162$ \\
\hline Parenting Relationship (PRS) & & & \\
\hline Attachment & 58 & 9.5 & $44-73$ \\
Communication & 55 & 10.9 & $39-66$ \\
Discipline Practice & 54 & 8.8 & $41-67$ \\
Involvement & 61 & 13.9 & $41-75$ \\
Parenting Confidence & 53 & 9 & $42-68$ \\
Satisfaction with Child's School & 57 & 11.3 & $38-68$ \\
Relationship Frustration & 60 & 8.2 & $36-64$ \\
\hline
\end{tabular}

\section{Focus group responses}

The audio taped focus group responses were transcribed by a professional transcriptionist and coded using Frame Work Analysis (FRA) as a coding guide. FRA is an iterative process which involves reviewing transcripts, generating overarching themes emerging from the focus group discussions, operationalizing those themes and evaluating the transcripts for exemplars of the themes. The investigators served as the primary coders for the transcripts. The following paragraphs report the common themes that emerged from the focus group discussions. Twelve themes emerged from the focus group discussions.

\section{What are their parenting challenges?}

There were four distinct themes that captured the participant's discussions of their parenting challenges. Two of those themes reflected the mothers' beliefs in the compatibility of their drug use with their role as a parent (i.e. Ability to parent even as they were abusing drugs and alcohol); and reflected their fears about enabling addictive behaviors in their children Two themes captured their struggles with mothering from prison and their recognition of the need to rebuild and reconstruct relationships with their children. Examples of the themes are presented below.

\section{"Parenting children with ADHD and medication issues"}

"My ten year old was diagnosed with ADHD at the age of five and they put him on narcotic medicine. I know he takes it while he goes to school and that's the only time he takes it, because if, if he. I don't want him to become addicted to it and it and it's an addictive drug."

"Mine had more than ADHD. It's ADHD, Bipolar and global developmental delays from birth defects."

\section{"Parenting from a distance"}

"It's hard to be an active parent if you're not with them."

\section{"Parenting in the midst of addiction"}

"I mean, a lot of times when people will talk to us they think that just because we have an addiction that we didn't parent our kids in the right way, and my kids, I don't agree with that, my kids always have mostly have what they wanted. You know, even an addict can put aside their issues to take care of their kids. Even the active part of addiction, turning that aside."

\section{“Losing children's trust and rebuilding relationships"}

"I kind of felt like I lost a little bit of their trust or something, you know because of my selfishness had taken me away from them... The older ones are not stupid, they know. "

I've had my oldest son tell me that he is proud of me for being here."

"I was afraid to ask my kids was their happiness affected. I never got the courage to ask. I was afraid they would be honest and it would be something hurtful."

\section{What are their parenting goals?}

Two themes captured the participant's parenting goals; being a good provider and a being a good mother. It is not uncommon for incarcerated mothers, especially those preparing to re-enter to talk about being a provider and being a good mother upon re-entering, as for some motherhood is central to their identity ${ }^{16}$. However, our participants also expressed a need to redefine their relationships with their children, as if to create a "new start point" in the mother child relationship. Examples of the themes are illustrated below.

\section{"Being a provider"}

"Meeting their basic needs and necessities; look back on their childhood and have good memories."

\section{"Being a good mother"}

"A mother, a good role model. Somebody that my children can talk to, I want to raise happy children."

\section{What are their perceptions of their social support?}

Two themes captured their discussions of their social support; fragile support and empowered support. Many of our participants acknowledged the importance of family support for maintaining sobriety; however for some family support represents something that was broken and will take much effort to repair. On the other hand, others stated they have a groundswell of enthusiastic family support upon reentry. Examples are presented below.

\section{"Fragile family support"}

"I envy all of you in here who have do have so you say, that you will have family support. I know you need family support to be able to make it from relapse and if you don't have it that makes it much harder almost set up for failure to relapse. " 
"It takes a toll on the family; it's not only you that you are hurting but your whole family."

"My family does not understand why I have not learned my lesson. They don't understand addiction."

\section{"Empowered family support"}

"My husband is supportive, so I mean that's a good thing I feel."

"The kid's dad, the kids, my mom, my sisters, everybody's going to be there for me. I am blessed to have them."

\section{How are they preparing for re-entry?}

Our participants are focused on transforming themselves as they prepare to re-enter. The themes reflected one of two ways in which they are accomplishing this transformation. Some are changing their basic characters defects by changing their thinking or Revisioning One's Self. Others are going inward, and engaging is a process of Self- Reflection. Examples of the themes are described below.

\section{"Re-visioning one's self"}

"I'm working through changing my thinking. You know it's all about whom I am and you know when you are working through your addiction because it totally changes the person that you are; changing my character defects."

\section{"Self reflection"}

"You have to look in... and you have to look at a lot of things that you have done. It's hard and challenging."

"I've been on the streets for a long time and it is hard. I keep doing the same thing over and over and I am getting tired of it."

"I found myself looking forward to when I got my last DUI. Looking forward to going to jail, because I knew it was the only way I was going to stop because I could not do it on my own."

\section{Have programs prepared them for reentry?}

Two themes captured their discussions of how programs have prepared them for re-entry. For some, while they acknowledge some form of job preparation assistance, they describe it as limited and they must pursue finding jobs on their own without assistance. Others were able to articulate specific program support, and those reflections were on the quality of the parenting programs offered. Examples of the themes are described below.

\section{“Limited program support"}

"I mean, we do have somebody who comes in and shows us how to, you know, do our interviews and do our, you know talk to people and things like that. I think that does help, but other than that, we're pretty much, other than the one specific person, we're pretty much on our own job seeking."

\section{"Specific Program Support"} helped."

"The parenting program that I did was creative and I think it

"I went to a parenting program at another facility and I learned a lot."

While the findings of the questionnaire data indicate that these mothers perceive that they have few challenges with parenting relationships, and they report their family and personal resources are adequate, the data from the focus groups provide deeper insight into their specific concerns and challenges with their children and their preparation for reentry.

Their articulated parenting goals as expressed in the focus group discussions align with those of other AOD dependent mothers. ${ }^{17}$ However, their challenges differ from those mothers in that they actively acknowledge and are aware of the need for relationship rebuilding and relationship repair especially with their children and their families. Furthermore, preparation for re-entry for some is an active cognitive process and social cognitive process (i.e. perspective taking), similar to the self-transformative process identified by Celinska and Siegel. ${ }^{16}$

\section{Discussion}

The goal of this exploratory project was three fold. Our first goal was to determine the parenting challenges and parenting goals of incarcerated AOD dependent mothers in recovery preparing to re-enter back into their families and communities. Our second was to obtain information on their social support, family and personal resources and their evaluation of the quality of those resources. Our last goal was to determine how well prepared they felt for re-entry and their perceptions of the effectiveness of their re-entry preparation.

To this end, we administered the mothers a series of questionnaires designed to tap their parenting stress, mental health, family and personal resources and their relationship challenges. We also engaged the mothers in focus groups discussions to further probe them about parenting challenges and goals, relationships, and reentry preparation.

Our central findings suggest the following. One, consistent with other research we observed that the mothers in our study experienced a variety of mental health and physical health challenges common to AOD women. ${ }^{11}$ Their reported history of physical and sexual abuse is also consistent with the findings from the extant literature on AOD women. ${ }^{18}$

While many of the mothers in our study reported currently receiving medication for their mental health problems, few reported receiving individual psychotherapy or participating in group therapy. It may be the case that in order to ensure their continued sobriety, and prevent relapse some form of individual and group therapy are needed once they renter back into their families and communities. Without quality treatment that addresses their on-going mental health needs and challenges, especially the lingering trauma associated with early physical and sexual abuse, the majority of these women will most likely relapse, and at some point in time return to prison. Furthermore we argue that untreated mental health problems comprise their ability to efficiently parent.

Second, the parenting challenges described by the mothers in our study during the focus group discussions were similar to the parenting challenges observed by Hardesty and Black ${ }^{3}$ in their research on AOD mothers (i.e. role model, provider). However, unlike the mothers in the Hardesty and Black study, our mothers were more explicit in identifying one specific parenting concern "parenting children on medication and with disabilities." The fact that they voiced this as a major parenting challenge could be interpreted in several ways. On the one hand this may indicate a "cry for help" or alternately it may suggest that they recognize their limitations in their ability to effectively parent children on medication and with developmental disabilities. The way in which they successfully negotiate this particular parenting challenge will depend in part on the availability of support structures and systems, and in part on their ability to avoid 
relapse. Empirical evidence strongly indicates that family support and program support are the most effective safeguards in preventing relapse and rearrest for AOD mothers. ${ }^{19}$

Third, with the exception of the mother's scores on the CESD, we urge caution in interpreting the results of the questionnaire data. Their responses to the questionnaires indicate that they have few parenting challenges, have adequate social and family support, and experience little parenting stress. We speculate that their responses may be more reflective of their perceptions of an idealized parenting role and romanticized notions of family support rather than reflective of their true parenting issues and family support issues.

We offer the following suggestions for future research and program design for this population of mothers. Ideally future research in this area should be planned to explore the following questions. How do parenting concerns differ across the stages of recovery and across the developmental ages of children? What are the most common parenting mistakes made by parents in recovery? What are the challenges/ successes inherent in rebuilding parenting role relationships? What are the multiple levels of support in place or created to sustain sobriety, and parenting? What are the "voices" of their young children and adult children in this process?

In terms of designing programs for re-entering AOD mothers. Dealing with parenting in addition to maintaining sobriety warrants multiple and continued levels of support, ${ }^{6}$ as maintaining sobriety may compete with maternal responsibilities. ${ }^{19}$ Parenting programs aimed at AOD dependent incarcerated mothers preparing for re-entry should be structured to provide targeted parenting support. More specifically these programs should be designed to address their real life issues and concerns in parenting post release (i.e. reconnecting with children and families, child mental and physical health issues), and to assist them in identifying triggers to prevent relapse in the context of parenting. Lastly we conclude that pathways back to parenting may differ for formerly incarcerated recovering AOD dependent mothers as recovery for some is a lifelong process and involves rebuilding a host of family relationships and supports overtime.

\section{Acknowledgments}

None.

\section{Conflicts of Interset}

None.

\section{References}

1. Inciardo J, Lockwood D, Potiger A. Women and Crack Cocaine. Macmillan Publishing, New York, USA. 1993.

2. Maher L. Sexed Work. Clarendon Press, Oxford. 1997.
3. Hardesty M, Black T. Mothering through addiction: a survival strategy among Puerto Rican Addicts. Qual Health Res. 1999;9(5):602-619.

4. Baker PL, Carson A. "I Take Care of My Kids" mothering practices of substance abusing women. Gender \& Society. 1999;13(3):347-363.

5. White W, Arria A, Moe J. Parenting in the context of addiction recovery: Critical research questions. Posted ar www.williamwhitepaers.com and www.facesandvoicesofrecovery.com. 2011.

6. Hammer A, Sanderson J, Mertin P. Influence of negative childhood experiences on psychological functioning, social support and parenting from mothers recovering from addiction. Child Abuse Negl. 1999;23(5):421-433.

7. Henderson DJ. Drug Abuse and incarcerated women: a research review. J Subst Abuse Treat. 1998;15(6):579-587.

8. Niccols A, Milligan K, Sword W, et al. Integrated programs for mothers with substance abuse issues: A systematic review of studies reporting on parenting outcomes. Harm Reduction Journal. 2012;9:14

9. O'Brien P. Maximizing success for drug affected women after release from prison. Women \& Criminal Justice. 2006;17(2/3):95-113.

10. Peugh J, Belenko S. Substance involved women inmates: challenges to providing effective treatment.The Prison Journal. 1999;79(1):23-44.

11. Clark BS, Rapkin K, Busen NH, et al. Nurse practitioners and parent education: a partnership for health. $J$ Am Acad Nurse Pract. 2001;13(7):310-316.

12. Harris YR, Graham JA, Carpenter-Oliver GJ. Children of incarcerated parents: theoretical, developmental and clinical implications. Springer Publishing Company, New York, USA. 2010;1-34.

13. Michalsen V. A Cell of One's Own? incarceration and other turning points in women's journey to desistance. Int J Offender Ther Comp Criminol. 2013;XX(X):1-20.

14. Few-Demo AL, Arditti JA. Relational vulnerabilities of incarcerated and reentry mothers: Therapeutic Implications. Int J Offender Ther Comp Criminol. 2013; XX(X):1-24.

15. Gil-Garcia JR, Pardo TA. Multi-method approaches to understanding the complexity of e-government. International Journal of Computers, Systems and Signals. 2006;7(2):3-17.

16. Celinski K, Siegel J. Mothers in trouble: coping with actual or pending separation from children due to incarceration. The Prison Journal. 2010;90(4):447-474.

17. Hanlon TE, O'Grady KE, Bennett-Sears T, et al. Incarcerated drug abusing mothers: their characteristics and vulnerability. Am J Drug Alcohol Abuse. 2005;31(1):59-77.

18. Kelley SJ. Stress and coping behaviors of substance abusing mothers. $J$ Soc Pediatr Nurs. 1998;3(3):103-110.

19. Robbins C, Martin S, Surratt H. Substance abuse treatment anticipated maternal roles, and reentry success of drug involved women prisoners. Crime \& Delinquency. 2007; 55(3):388-411. 